\title{
Secondary carcinoma of left atrium simulating
}

\section{myxoma}

\author{
A C THOMAS, $P$ G Mills,* $N$ M GiBBS, $M$ J DAVIES \\ From the Departments of Histopathology and Cardiology, St George's Hospital Medical School, \\ London, and the Department of Histopathology, St Luke's Hospital, Guildford, Surrey
}

SUMMARY Three cases are presented in which secondary carcinoma in the left atrium mimicked atrial myxoma or infective endocarditis. Constitutional features of weight loss, fever, anaemia, leucocytosis, raised erythrocyte sedimentation rate, and systemic emboli were prominent clinical features. The association of these systemic manifestations with both secondary deposits in the atrium and atrial myxomata may be the result of an immune response to circulating tumour antigens. Mitral stenosis was present in two of the three patients suggesting that left atrial thrombus may provide a site for the seeding of malignant cells.

In patients dying with disseminated secondary carcinoma a detailed and specific search of the heart at necropsy shows that between 5 and 25 per cent have cardiac deposits. ${ }^{1-3}$ The most frequent morphological patterns seen are diffuse pericardial deposits and one or more intramyocardial nodules, ranging in size from microscopical foci to those several centimetres across. In contrast to the relatively common pathological finding of cardiac secondaries, significant clinical symptoms or signs are rare. Characteristic clinical presentations include cardiac tamponade, ${ }^{34}$ constrictive pericarditis, ${ }^{4}$ atrial arrhythmias, ${ }^{3}$ conduction disturbances, ${ }^{5}$ and obstruction to right ventricular outflow. ${ }^{6}$ Systemic manifestations of fever, a high erythrocyte sedimentation rate, weight loss, anaemia, and abnormal serum proteins are phenomena usually associated with atrial myxomata, ${ }^{78}$ and may be found both with and without systemic emboli. We describe three cases in which these systemic manifestations were the presenting features of left atrial deposits from carcinomata.

\section{Case reports}

\section{CASE 1}

A 72-year-old woman was admitted complaining of breathlessness for two years and sudden loss of movement in the left arm and leg. She was in atrial

* Present address: The Cardiac Department, The London Hospital, Received for publication 23 May 1980 fibrillation with clinical signs of pulmonary hypertension, a soft opening snap, and a grade $2 / 4$ middiastolic murmur. A dense left hemiparesis was noted but there was no splenomegaly, no splinter haemorrhages, and no evidence of emboli in the optic fundi. Investigation showed: $\mathrm{Hb} 11.3 \mathrm{~g} / \mathrm{dl}$; a leucocytosis of 26800 ; ESR $92 \mathrm{~mm} / \mathrm{h}$; serum Ig $G, A$, and $M$ all normal; urine analysis, 20 $\mathrm{RBC} / \mathrm{mm}^{3}$; electrocardiogram, $\mathrm{QRS}$ axis $+90^{\circ}$ and partial right bundle-branch block. A chest $x$-ray film showed left atrial enlargement with dilated pulmonary veins. An echocardiogram showed left atrial dilatation and typical rheumatic mitral stenosis, with no evidence of left atrial myxoma.

Diagnoses of rheumatic mitral stenosis and cerebral emboli were made. She was treated with anticoagulants and the ventricular rate was controlled with digoxin. The erythrocyte sedimentation rate and total white count showed no change and 20 days after admission a profuse crop of splinter haemorrhages appeared in the left hand and a soft pansystolic murmur was noted. Despite persistently negative blood cultures and a sterile marrow aspirate, a diagnosis of bacterial endocarditis was made. Broad-spectrum antibiotic therapy was initiated, but had no effect, and the patient died.

Necropsy showed moderate rheumatic mitral stenosis (valve orifice $1.8 \mathrm{~cm}^{2}$ ). The left atrium was partially occupied by a layer of pale friable thrombus containing abundant malignant cells (Fig. 1). The left adrenal contained a necrotic tumour, $2.5 \mathrm{~cm}$ in diameter, extending into the perirenal fat. Cerebral, 
Fig. 1 Case 1: View of left atrium from above showing friable mural thrombus. Histology showed the presence of numerous malignant cells.

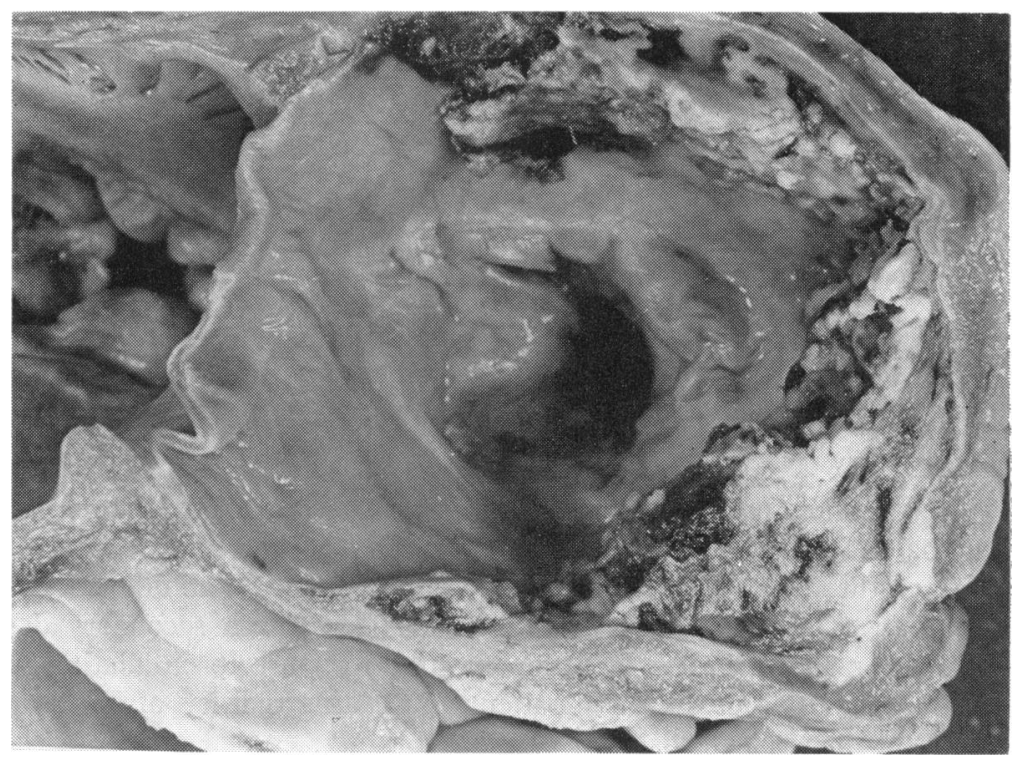

renal, and splenic emboli consisted largely of tumour cells. Electronmicroscopy showed neurosecretory granules in isolated tumour cells suggesting a primary adrenal medullary tumour.

\section{CASE 2}

A 76-year-old woman gave a history of rheumatic fever at the age of 35 . She had presented at the age of 74 with severe dyspnoea and was found to have tight mitral stenosis. Mitral valvotomy was carried out and her dyspnoea was dramatically improved. Sixteen months later, however, she became generally unwell with a persistent low grade pyrexia and weight loss. There were persistent and unchanging physical signs of mild mitral stenosis and regurgitation. Investigations showed: ESR greater than $120 \mathrm{~mm} / \mathrm{h} ; \mathrm{Hb} 8.6 \mathrm{~g} / \mathrm{dl}$; total white blood cell count 26000 ; alpha-2-globulin increased; fibrinogen increased to $8.3 \mathrm{~g} / \mathrm{l}$. Though the patient continued to lose weight and remained pyrexial, blood cultures were persistently negative. An echocardiogram showed a left atrial dimension of $6.3 \mathrm{~cm}$ but no detectable myxoma.

Necropsy showed a tumour mass $4.0 \mathrm{~cm}$ in diameter within the interatrial septum and eroding into the left atrium where it was covered by thrombus. The mitral valve showed chronic rheumatic valve disease with scarring from a previous valvotomy (orifice $2.5 \mathrm{~cm}^{2}$ ). A $6 \mathrm{~cm}$ tumour mass was found within the left adrenal extending into perirenal fat. No other tumour deposits were found and the histology suggested a primary malignant adrenal tumour. Electron- microscopy did not show secretory granules within tumour cells and there was insufficient other evidence to classify the tumour as medullary or cortical.

\section{CASE 3}

A 38-year-old woman presented with sudden onset of a mild left hemiplegia, chest pains, and breathlessness. Three years previously she had been treated for stage IIb carcinoma of cervix by radiotherapy. Two weeks after admission to hospital a blotchy skin rash and subungual splinter haemorrhages appeared. Investigations on initial admission showed: $\mathrm{Hb} 14.1 \mathrm{~g} / \mathrm{dl}$; total white cell count 16400 ; ESR $60 \mathrm{~mm} / \mathrm{h}$. She remained apyrexial throughout. A chest $x$-ray film showed shadowing in the left upper lobe. Lower limb phlebograms did not show venous thrombosis and a lung scan was normal. The clinical diagnoses considered were pulmonary emboli and bacterial endocarditis but blood cultures and serological tests for brucellosis and psittacosis were negative. The patient deteriorated and in view of the previous history of carcinoma and persistent lung shadow it was felt that disseminated malignancy was likely.

Necropsy showed pulmonary lymphatic tumour permeation, metastatic tumour in paracolic, coeliac, and hilar lymph nodes, and small hepatic deposits. The heart was normal apart from a rounded mass, measuring $2 \times 1.5 \mathrm{~cm}$, firmly attached to the centre of the atrial surface of the anterior cusps of the mitral valve. The underlying cusp was slightly thickened but with no destruction of valve tissue. 


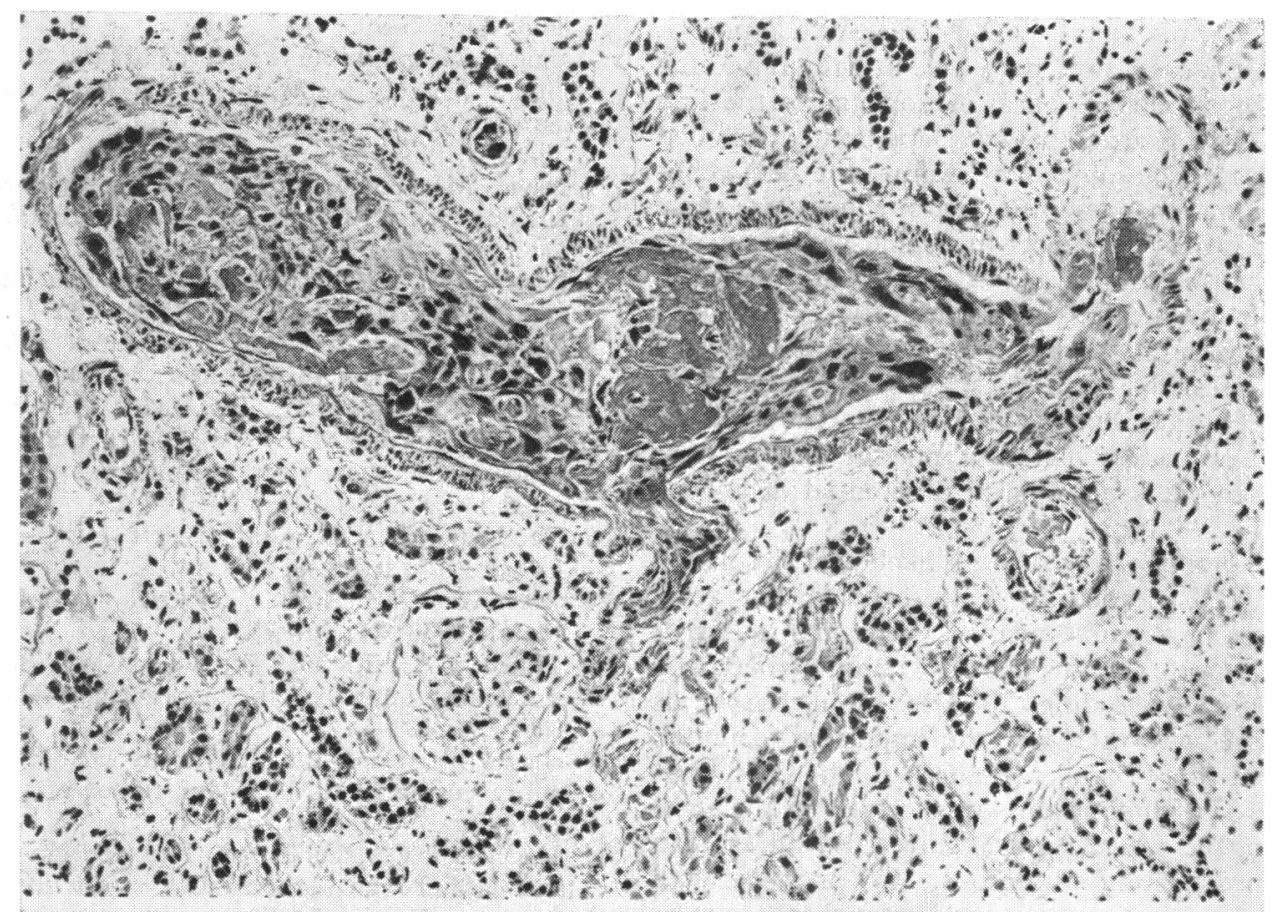

Fig. 2 Case 3: Photomicrograph showing tumour embolus in a medium sized renal artery.

Histologically the mass consisted of tumour cells with a superficial covering of thrombus. The kidneys showed one area of infarction which was the result of tumour embolus (Fig. 2).

\section{Discussion}

In necropsy series the frequency of myocardial or pericardial secondaries may be as high as 25 per cent because of selection. ${ }^{2}{ }^{3}$ Even in retrospect, however, the majority of patients with cardiac secondaries have no recognisable clinical symptoms or signs. Thus in 302 patients with cardiac secondaries, ${ }^{3}$ only six had related symptoms, three with pericardial tamponade caused by malignant effusions and three with atrial tachyarrhythmias associated with intra-atrial tumour deposits.

Glancy and Roberts ${ }^{4}$ in a retrospective study of the clinical data of 70 patients with disseminated malignant melanoma could not separate those with and those without cardiac metastases. Thurber and his colleagues ${ }^{9}$ reviewed the electrocardiograms of 148 patients who had cardiac secondaries; 48 were abnormal, but in none was the abnormality clearly related to the secondary deposits. Berge and Sievers ${ }^{1}$ also failed to show any specific electro- cardiographic abnormalities in patients with cardiac secondaries. In some instances, however, myocardial metastases have been shown to produce electrical abnormality simulating ischaemic damage. ${ }^{10}$ Thus, the great majority of cardiac metastases are clinically silent and the general systemic manifestations and embolic phenomena found in our three patients have not been previously emphasised.

Systemic manifestations including fever, high erythrocyte sedimentation rate, raised white blood cell count, and increased serum globulins are usually regarded as characteristic of left atrial myxomata and were found in varying combinations in 40 of 45 patients with this condition. ${ }^{7}$ The mechanism of these systemic phenomena is obscure $^{8}$ but has been linked to either necrosis within the myxoma ${ }^{11}$ or an immune response to tumour antigens. ${ }^{12}{ }^{13}$ In the three cases presented here systemic manifestations were associated with secondary carcinoma in the left atrium, and this supports the theory that tumour cells within thrombi in the systemic circulation may be a potent stimulus for an immune response to tumour antigens. Pure thrombus in the left atrium, commonly seen in chronic rheumatic disease, is not associated with such a clinical picture. 
The systemic manifestations and embolic phenomena in the cases presented here initially raised the clinical possibility of atrial myxomata. In the first two cases a left atrial mass was apparently excluded by echocardiography. The failure of the echocardiograms to show the tumour mass in these patients probably reflects its sessile nature and its fixation to the atrial wall. After left atrial myxoma, infective endocarditis remained the most likely clinical diagnosis despite negative blood cultures. A case of left atrial metastases from an unrecognised ovarian choriocarcinoma presenting with anaemia, leucocytosis, and a high erythrocyte sedimentation rate posed the same clinical differential diagnostic problems. ${ }^{14}$

In the first two cases it seems likely that circulating tumour cells seeded in a pre-existing left atrial thrombus and subsequently grew into a larger tumour mass. The presence of chronic rheumatic valve disease leading to left atrial thrombus formation was therefore an important common factor in these two patients. In the last case, however, the tumour mass was located on the atrial aspect of the anterior mitral valve cusp rather than in a pre-existing thrombus. In patients with disseminated malignancy, non-bacterial endocarditis is not an uncommon finding. If these marantic (non-bacterial thombotic endocarditis) vegetations are sectioned a tiny proportion is found to contain malignant cells. It seems possible that in our third patient this process provided the nidus for tumour growth, a process which has been aptly named malignant endocarditis. ${ }^{15}$ Microscopical malignant vegetations may be more common than is usually realised but probably few patients live long enough to develop a large tumour mass.

\section{References}

1 Berge T, Sievers J. Myocardial metastases. A pathological and electrocardiographic study. Br Heart $\mathcal{f}$
1968; 30: 383-90.

2 Fine G. Neoplasms of the heart and pericardium. In: Gould SE, ed. Pathology of the heart and blood vessels. 3rd ed. Springfield, Illinois: Charles C Thomas, 1968: 851-83.

3 Davies MJ. Tumours of the heart and pericardium. In: Pomerance A, Davies MJ, eds. The pathology of the heart. Oxford: Blackwells, 1975:

4 Glancy DL, Roberts WC. The heart in malignant melonama. Am f Cardiol 1968; 21: 555-71.

5 Redwine DB. Complete heart block caused by secondary tumors of the heart. Case report and review of the literature. Tex Med 1974; 70: 59-64.

6 Griffith DNW, Myers A. Obstruction of right ventricular outflow tract by solitary ovarian metastases. Br Heart f 1978; 40: 700-2.

7 Goodwin JF. Diagnosis of left atrial myxoma. Lancet 1963; i: 464-8.

8 Greenwood WF. Profile of atrial myxoma. Am $\mathcal{F}$ Cardiol 1968; 21: 367-75.

9 Thurber DL, Edwards JE, Achor RWP. Secondary malignant tumors of the pericardium. Circulation 1962; 26: 228-41.

10 Harris TR, Copeland GD, Brody DA. Progressive injury current with metastatic tumor of the heart. Am Heart f 1965; 69: 392-400.

11 MacGregor GA, Cullen RA. The syndrome of fever, anemia and high sedimentation rate with an atrial myxoma. $\mathrm{Br}$ Med f 1959; ii: 991-3.

12 Boss JH, Bechar M. Myxoma of the heart. Am $\mathcal{f}$ Cardiol 1959; 3: 823-8.

13 Currey HFL, Mathews JA, Robinson J. Right atrial myxoma mimicking a rheumatic disorder. $\mathrm{Br} \mathrm{Med} \mathcal{F}$ 1967; i: 547-8.

14 Hepp A, Larbig D, Bader H. Left atrial metastasis of chorion carcinoma, presenting as mitral stenosis. Br Heart f 1977; 39: 1154-6.

15 Coller FC, Inkley JJ, Moragues V. Neoplastic endocardial implants. Am $\mathcal{f}$ Clin Pathol 1950; 20: 159-64.

Requests for reprints to Professor M J Davies, Department of Histopathology, St George's Hospital Medical School, Cranmer Terrace, London SW17. 